\title{
Vestibular contributions to high-level sensorimotor functions
}

\author{
W. Pieter Medendorp*, Luc J.P. Selen \\ Radboud University, Donders Institute for Brain, Cognition and Behaviour, P.O. Box 9104, NL-6500 HE, Nijmegen, The Netherlands
}

\section{A R T I C L E I N F O}

\section{Keywords:}

Vestibular cognition

Optimal control

Bayesian

Decision making

Visual stability

Motor learning

\begin{abstract}
A B S T R A C T
The vestibular system, which detects motion and orientation of the head in space, is known to be important in controlling gaze to stabilize vision, to ensure postural stability and to provide our sense of self-motion. While the brain's computations underlying these functions are extensively studied, the role of the vestibular system in higher level sensorimotor functions is less clear. This review covers new research on the vestibular influence on perceptual judgments, motor decisions, and the ability to learn multiple motor actions. Guided by concepts such as optimization, inference, estimation and control, we focus on how the brain determines causal relationships between memorized and visual representations in the updating of visual space, and how vestibular, visual and efferent motor information are integrated in the estimation of body motion. We also discuss evidence that these computations involve multiple coordinate representations, some of which can be probed in parietal cortex using neuronal oscillations derived from EEG. In addition, we describe work on decision making during self-motion, showing a clear modulation of bottom-up acceleration signals on decisions in the saccadic system. Finally, we consider the importance of vestibular signals as contextual cues in motor learning and recall. Taken together, these results emphasize the impact of vestibular information on high-level sensorimotor functions, and identify future directions for theoretical, behavioral, and neurophysiological investigations.
\end{abstract}

\section{Introduction}

Cognition is the collective term for the processes and mechanisms that produce intelligent behavior (Chemero, 2009; Clark, 2010). A hallmark of intelligent behavior is the coupling between perception and action. Actions are achieved by activating our motor system: we look, reach or turn our heads. Some scientists are convinced that our perceptions, thoughts and memories only exist to organize our actions (Tweed, 2003; Wolpert et al., 2001). In this view, cognition is there to establish the link between sensory input and motor output, a process often referred to as sensorimotor integration, and higher order cognitive functions could only evolve because of our action capabilities. Thus, in turn, by studying sensorimotor integration we also open a window on cognition. For example, how we integrate the past in the present, or how we make decisions in a world full of action possibilities, or how rewards have an effect on motor learning.

In the seventeenth century, the French philosopher and mathematician René Descartes already described the connection between input, i.e. sensation, and the output, i.e. motor behavior (Descartes, 1644). However, he did not have the techniques available to unravel the complex neurocognitive link between the two. History still had to walk a long way, which took us through the nineteenth-century phrenology, the forerunner of neuropsychology, via the neuron doctrine, to arrive at neural circuits and systems levels perspectives (Yuste, 2015).

We now know that sensory and motor signals are associated with context, memories of earlier experience, and knowledge. This enables us to achieve a coherent perception of the world and plan, anticipate, and decide on appropriate actions to change the state of the world to our benefit. At the perception level, the brain has to infer or estimate the state of the world (e.g., where and what are interesting targets) and body (e.g., where are my hands), while at the action level it is concerned with prospective control, i.e. generating the motor commands needed to acquire a particular task goal. Recent mathematical insights based on optimality principles suggest that both the inference and control problem are intertwined in many ways (Todorov and Jordan, 2002; Todorov, 2010), in line with findings at the neural implementation level (Cisek and Kalaska, 2010).

While considerable advances have been made in understanding the component processes in stationary, impoverished environments - for example, how visual perception remains stable across eye movements, how sensory information affects response selection, or how stationary subjects assess the dynamics of the world - research is just beginning to address the neurocognitive computations contributing to perception and action while the body is in motion, a common daily situation. In this review, we mainly, but not exclusively, cover recent research related to perceiving and acting while in motion, particularly focusing

\footnotetext{
* Corresponding author.

E-mail address: p.medendorp@donders.ru.nl (W.P. Medendorp).
} 
on the role of the vestibular system in high-level sensorimotor functions.

We summarize studies examining the ability of healthy participants to make perceptual judgments, select responses, and learn multiple motor actions, especially when the body is in motion and effects of inertial forces and body-motion must be integrated into perceptual estimates and motor actions. First, we will provide a sketch of the general framework within which we will discuss the incorporation of vestibular signals into sensorimotor function. This framework involves concepts like optimization, inference, control, learning and coordinate representations, all of which will be used to guide this review.

\section{General framework}

Many theories in the sensorimotor domain build on the assumption that perception and action and their integration are in some way optimal, given the constraints imposed by the task, the sensory modalities and the motor apparatus involved.

Starting with our sensors, they provide noisy information that is often even ambiguous or redundant. For example, the vestibular sensors provide an estimate of the orientation of the head in space. However, an estimate of the head orientation in space can also be deduced by combining information about body orientation in space, based on somatosensory signals, with information about the head orientation relative to the body, derived from neck proprioception (Clemens et al., 2011). When there are redundant information sources, optimality suggest that the best estimate can be derived based on statistical inference, a process called multisensory integration. According to this notion, uncertainty in the internal estimates of the state of the body and world, caused by noise or ambiguity, can be reduced by integrating overlapping information from different sensory modalities, and weighting each signal in proportion to its reliability (Clemens et al., 2011; Ernst and Bülthoff, 2004; Körding and Wolpert, 2004). In the example above, the brain is supposed to derive the best estimate of head orientation in space by integrating the vestibular estimate with the combined estimate derived from neck-proprioception and body somato-sensation.

Like incoming sensory information, outgoing efferent control signals travelling to the peripheral motor system are corrupted by noise (Faisal et al., 2008), often depending on the strength of the signal (Jones et al., 2002). This imposes constraints on the optimal muscular activation pattern to attain the highest endpoint accuracy (Harris and Wolpert, 1998). At the motor level, we select the target and control the motor commands of the effectors to achieve a rewarding state, formulated explicitly in terms of gains and losses (Diedrichsen et al., 2010). For any possible action, we need to know the associated costs as well as the rewarding nature of the sensory states that it might achieve. This requires implicit knowledge about body and world dynamics, called a forward internal model (Angelaki et al., 2004; Kawato, 1999). The same internal model is used by the brain to predict the sensory consequences of the motor commands sent to the motor periphery, which is also mandatory to differentiate between sensations that arise as a consequence of one's own movements and those that arise from the environment (Berniker and Kording, 2008; Holst and Mittelstaedt, 1950; Sommer and Wurtz, 2008; Von Helmholtz, 1867). In order to keep sensory predictions accurate, the forward model must be continuously calibrated to the actual dynamics of body and world. The process responsible for this calibration is called motor adaptation (Shadmehr et al., 2010).

These optimality considerations for the sensorimotor system are theoretically reconciled in Optimal Feedback Control Theory (Diedrichsen et al., 2010; Shadmehr and Krakauer, 2008; Todorov and Jordan, 2002), which is graphically depicted in Fig. 1. In this model, the state estimator combines current sensory information with the predicted outcomes based on earlier motor outputs to infer a belief about the state of the world and body. These state estimates are fed into

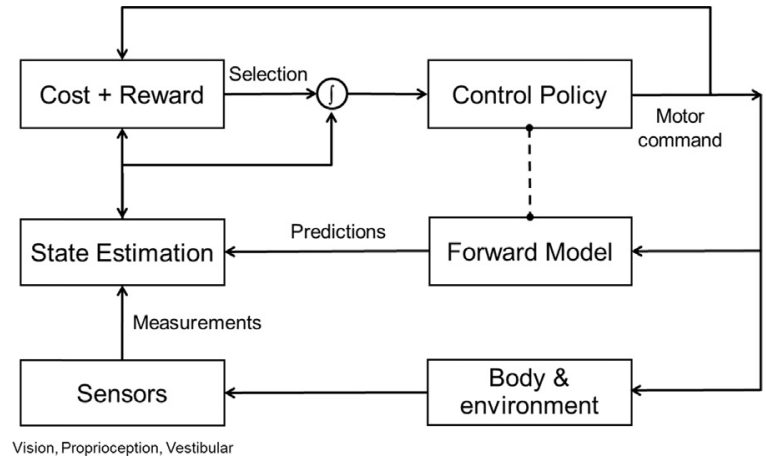

Fig. 1. Optimality as a framework for studying sensorimotor processes. Depicted is a graphical representation of an Optimal Feedback Control framework (Shadmehr and Krakauer, 2008; Todorov and Jordan, 2002). For the major part of this review we will focus on the state estimator that takes priors, often based on the forward model's predictions, and sensory inputs to estimate the state of the world and body in multiple reference frames. However, also cost and reward computations are considered in relation to vestibular inputs. We will further discuss how vestibular inputs may serve as a cue to select and update the appropriate control policies and forward models. The dashed line indicates that in motor adaptation paradigms both the control policy and forward model are updated. Based on the cost and reward structure, the brain needs to select on which target to act upon and with which effector.

a controller that executes a so-called control policy - a set of rules specifying how to act given the estimated current state of the body and world to attain the task goal. The control policy is derived based on the constraints imposed by sensory uncertainty, motor uncertainty and the relative importance of effort and accuracy.

This framework of optimal multisensory integration and optimal control policies has been tested extensively in stationary conditions, especially for looking and reaching while the head and body are immobilized. Only recently the validity of this framework has been addressed in more complex, real-life, conditions where the whole body is in motion. When the body is in motion, the number of contributing sensory and motor systems increases and they must be used in synergy in order to perceive, decide and act in relation to the multitude of possible and unpredictable events, in many alternative ways.

In the next sections we will discuss recent advances in our understanding of multisensory integration and control when there is, often conflicting, noisy information from which the state of the world and/or body must be derived.

\section{Causal inference in visual stability}

As shown in Fig. 1, the state estimator integrates current sensory information with the predicted outcomes based on motor outputs to infer the state of the world and body. Evidence for this computation is, for example, seen in updating the expected state of the visual world during a saccadic eye movement. Despite the continuous changes in visual input due to saccadic eye movements, our internal estimate, or representation, of the world remains stable. Behavioral and neurophysiological studies suggest that the brain predicts the changes in the retinal image induced by the saccade by using a copy of an eye's movement command, routed into a forward dynamic model (Medendorp, 2011; Sommer and Wurtz, 2008). The state estimator is supposed to update its beliefs about the state of the world by integrating this prediction signal with the actual, re-afferent, visual input.

Under the assumption that objects in the world do not change position during a saccade, optimal integration rules say that the predicted and actual sensory feedback should be integrated according to their reliability. Indeed, various studies have shown evidence for this idea (Munuera et al., 2009; Niemeier et al., 2007; Oostwoud Wijdenes et al., 2015; Vaziri and Shadmehr, 2006). For example, Niemeier et al. (2003) found evidence for such an optimal integration scheme using a 
paradigm that measures how well subjects detect changes of object locations across saccades. In this paradigm - called saccadic suppression of displacement - participants watch dots on a screen, and at the moment the eye moves, one of the dots jumped to a slightly different location. Participants are then asked to report the initial, pre-saccadic, perceived location of the displaced spot. Niemeier and colleagues found that the localization response contracted to the post-saccadic position of the dot, in a nonlinear relation with the size of the jump. They were able to explain these findings using an optimal integration model that combines noisy motor and visual signals with a prior belief that reflects the assumption that dots are not displaced during the saccade.

While such an optimal integration scheme results in a more precise representation of the dot's location, it goes at the expense of systematic biases when a dot jumps. In other words, the localization will always be pulled to the post-saccadic location of the dot, irrespective of whether the size of the displacement is small or large. However, it is well possible that for large displacements an inference can be made that its location does not represent the pre-saccadic location at all, and hence should be ignored to make an inference about the pre-saccadic location.

This latter notion implies that the brain has to estimate the causal relationship between initial pre-saccadic position, as stored in memory, and the position percept of the post-saccadic visual stimulus to establish to what degree they can be integrated or whether they should be kept segregated. This computation not only depends on the precision of the pre- and post-saccadic position signals, which may be affected by the time the stimuli have been viewed, but also on their spatial difference.

Recently, we modeled this aspect of state estimation through a Bayesian causal inference mechanism, in which at the trial level an optimal mixing of two possible strategies, integration vs. segregation of the pre-saccadic and the post-saccadic position signals, is applied (Atsma et al., 2016). This is referred to as a mixture model, which was originally used by Körding et al. (2007) to study audiovisual integration and segregation.

In the model by Atsma and colleagues, shown in Fig. 2A, the task of the optimal observer is to estimate the pre-saccadic target position (s) based on two sources of information, the memory-based pre-saccadic

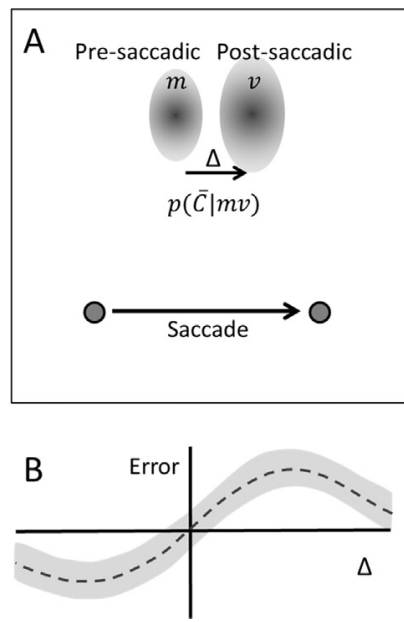

Fig. 2. Mixture model for visual stability across saccades. A stimulus is viewed before a saccade and briefly shown again after the saccade, but at a displaced position (indicated by $\Delta$ ). Based on the pre-saccadic $(m)$ and post-saccadic $(v)$ representations, represented by probability distributions (gray blobs), the probability of an unstable object, $p(\bar{C} \mid m, v)$, is computed. In case $m$ and $v$ are unrelated the best solution is to segregate and ignore $v$ when indicating the pre-saccadic stimulus location. If $m$ and $v$ derive from the same stimulus, the best solution is to integrate $v$ in the computation of the pre-saccadic location. The two solutions are weighted according to the probabilities that $m$ and $v$ are unrelated and related, respectively. B. The mixture model predicts localizations errors of the pre-saccadic stimulus location to become larger with increasing displacements $(\Delta)$ till a certain point, beyond which they transition back to smaller errors. position $(m)$ and the position of the post-saccadic visual stimulus $(v)$. Because both are noisy representations, they are represented by probability distributions. Depending on the discrepancy between these two sources of information (indicated by $\Delta$ ), the state estimator could infer no evidence for a displacement and consider the information provided by the post-saccadic stimulus, $v$, as relevant for its position estimate and thus to be integrated with the memory of the pre-saccadic target location to determine the pre-saccadic position $s$. The state estimator, however, could also infer that $v$ is a new visual object that is unrelated to $s$. This should evoke a segregation step, in which only the pre-saccadic location is taken into account. Thus the state estimator could distinguish two kinds of trials, which require different forms of $p(s \mid m, v)$ should be $p(s \mid m, v)$. From an optimality perspective, the state estimator should not choose per trial one of these forms, but should apply on any trial a mix of both, with the weight for each form equal to the estimated probability of it being consistent with the given sources of information $m$ and $v$.

If we indicate the situation of a trial by $C$ when there is a common cause for $m$ and $v$ and by $\bar{C}$ when $v$ derives from a different object, this leads to a mixture model of the representation of $p(s \mid m, v)$ :

$p(s \mid m v)=p(s \mid m, v, C) \bullet p(C \mid m, v)+p(\operatorname{sl} m, v, \bar{C}) \bullet p(\bar{C} \mid m, v)$

This equation comprises three components: (i) $p(s \mid m, v, C)$, the distribution of $s$ based on the integration of $m$ and $v$ when $v$ is the sensory representation of the true position; (ii) $p(\operatorname{sim}, v, \bar{C})$, the distribution of $s$ based on the segregation of $m$ and $v$ when $v$ represents a displaced version of the true position and (iii) $p(C \mathrm{~lm}, v)$, the probability that the current $m$ and $v$ are from a trial with common source, with $p(\bar{C} \mid m, v)=1-p(C \mid m, v)$ the complementary probability. Under the assumptions that the probability distributions are Gaussian, an analytic solution for $p(s \mid m, v)$ can be derived, also in two dimensions (see Atsma et al., 2016 for further details).

We tested the performance of this mixture model using an extended version of the saccadic suppression of displacement paradigm, predicting that localization errors become larger with increasing displacements, but beyond a certain displacement they transition back to smaller errors (Fig. 2B). Participants viewed three targets, with one of them the fixation point, the other the saccade target and a third peripheral target. After the saccade, one of the three targets remained, for different viewing durations, but often at a slightly displaced position, and participants had to indicate which location it had prior to the saccade. We found different localization errors for the three targets, depending on the viewing time of the post-saccadic stimulus and its spatial separation from the pre-saccadic location. The proposed mixture model could nicely account for these observations, thus suggesting that a causal inference mechanism is used by the state estimator in order to evaluate whether memorized and visual representations derive from a single stable object or not.

The subsequent question is how the state estimator performs these computations. Before we discuss potential answers to this question, we will first briefly describe work on state estimation of body motion.

\section{Multisensory integration in perception of body motion}

State estimation of body motion is based on inputs from various sensory modalities, including the vestibular, somatosensory and visual systems. In addition, when the motion is self-generated, efference copies of motor commands can also provide information about how far we move and in which direction we are heading.

The vestibular system, located in the labyrinth of the temporal bone in the inner ear, comprises two sensors, the semicircular canals and the otoliths, which detect angular velocity and linear acceleration, respectively. Since the otoliths are sensitive to both the pull of gravity and head accelerations, together called gravito-inertial force, they cannot distinguish between head tilt and head acceleration (Angelaki et al., 
1999). The disambiguation of the otolith signal into its acceleration and gravitational components depends on the canal signal (Laurens and Droulez, 2007; Laurens et al., 2013; Merfeld et al., 1999), but may also involve cognitive processes (Clemens et al., 2011; Wertheim et al., 2001).

The optokinetic system is a visual subsystem for motion detection based on optic flow. While the vestibular and optokinetic systems operate in different frequency domains, there is good evidence that the brain integrates their information when navigating through the environment (Gu et al., 2008). Several behavioral and neurophysiological studies have shown that the uncertainty in the internal estimate of heading (i.e. the direction of self-motion) is reduced by weighting each signal in proportion to its reliability, even when cue reliability varies unpredictably across trials (see Fetsch et al. (2013) for review).

Displacement estimation also relies on vestibular and optokinetic information, but there are several complicating factors when deriving a displacement estimate instead of a heading estimate from them. For example, optic flow signals can directly be used to estimate heading (Gibson, 1966), but require depth scaling to provide a cue for displacement estimation (Frenz and Lappe, 2005). Furthermore, heading estimation improves through evidence accumulation with the ongoing motion (Drugowitsch et al., 2014), which cannot be the case in displacement estimation. In other words, displacement estimation requires the integration of sensory information across the whole motion, i.e., from start to end.

Recently, we showed that human observers optimally combine visual and vestibular information during a displacement estimation task (ter Horst et al., 2015). In our experiments, participants were translated using a linear sled, which was immersed in a stereoscopic virtual reality environment that provided optic flow patterns associated with the motion. Subjects were exposed to different levels of visual coherence to change relative cue reliability and different discrepancies between the optokinetic and vestibular cue. Results show that humans estimate their body displacement by adapting their weighting of visual and vestibular information from trial to trial in proportion to their reliability.

Furthermore, during body motion, the eyes typically also move to maintain fixation on objects of interest. While the brain uses these eye movement signals to factor out the optic flow component related to self-motion (Warren and Hannon, 1990), eye movements could also directly affect the perception of self-motion, even in the absence of optic flow. In a recent study, we asked human subjects to estimate their displacement when the motion was accompanied by fixation on a world-fixed, or on a body-fixed fixation point or without a fixation point at all (allowing free gaze). Our results show that extra-retinal eye movement signals are used as a cue in the perception of body motion, weighting for about $25 \%$ into the percept, even in the absence of optic flow (Clemens et al., 2017). This novel result implies that any study concerned with self-motion estimation cannot ignore the possible influence of eye movements.

The somatosensory system may also detect body motion, by signaling the changing pressures on the skin and the proprioceptive signals that provide information about the relative position of body, head, and eyes. It has even been argued that internal, visceral signals play a role in detecting self-motion (Mittelstaedt, 1992; Trousselard et al., 2004). Integrating these sensory signals in an appropriate fashion is crucial for state estimation, but involves complex computations, not only taking into account differences in reliability, but also their different dynamics and coordinate representations. In the next section, we will consider the reference frames and coordinate representations involved in state estimation and updating across whole body motion.

\section{State estimation based on multiple coordinate representations}

Because information that arrives at the state estimator could have different coding formats and reference frames, an important question concerns the coordinate representations that are used to encode the state estimate. These coordinate frames can be linked to neurons, or neural maps, that the brain uses to organize its information processing, including eye-centered, head-centered, body-centered or intermediate frames of reference. Neurons with eye-centered receptive fields have been implicated in state estimation across saccades. Target representations coded by these neurons are predictively updated based on an efference copy of the eye movement command (Sommer and Wurtz, 2008). When the input is visual, representations in other reference frames, for example body-centered representations, can also be constructed from the initial eye-centered representation, taking eye and head position into account.

While spatial representations in body coordinates can be considered invariant to eye movements, this is not the case for body movements. When the body moves, these body-centered representations are also no longer in register with external space, unless they are actively updated. Recently, we addressed the question of whether the state estimator updates the representations in the various reference frames simultaneously, or whether it relies on a single frame for updating, from which representations in other reference frame are subsequently constructed (Tramper and Medendorp, 2015).

For simplicity, we considered only two reference frames in the coding of state estimates, an eye- and a body-centered frame, but our reasoning below can be extended to the whole heterogeneous collection of reference frames that is found in the brain. We implemented an optimal integration model that mimics the updating of state estimates during passively-induced body translations in combination with eye rotations. In this model, shown in Fig. 3A, a visual scene is initially stored in an eye-centered map and transformed into a body-centered map. During the motion, the sensory systems provide a noisy estimate of the body displacement (see preceding paragraph). Crucially, because the noise on this signal is direction-specific (i.e., the uncertainty will be largest along the axis of body motion), it will affect the update of the eye-centered and body-centered representations differently for different target directions in space (see Fig. 3B). The model integrates these two spatial estimates according to their reliability, which involves a reference frame transformation, to yield the estimate of the scene after updating. For comparison we also modeled the state updating in either an eye-centered or body-centered reference frame.

To test these models, we designed an experiment in which participants had to remember the location of a briefly presented target while being translated sideways. The behavioral responses were in agreement with the model that uses a combination of eye- and bodycentered representations, weighted according to the reliability of the representation after the updating. These findings suggest that the brain simultaneously updates representations in multiple reference frames across body motion. Because these representations are kept in sync, they can be optimally combined to provide a more precise estimate of visual locations in space than based on single-frame updating mechanisms. An analog conclusion was recently reported for reaching movements to visuo-proprioceptive targets (McGuire and Sabes, 2009). We believe that this can be generalized to the whole collection of reference frames.

Support for our conclusion also comes from animal work, showing that parietal cortex receives both the vestibular signals (Shinder and Taube, 2010) and eye position signals (Prevosto et al., 2009), which can be integrated to compute a self-motion estimate. This estimate could be used to update state estimates in a mixture of reference frames, including eye-centered, body-centered and, intermediate coordinates 

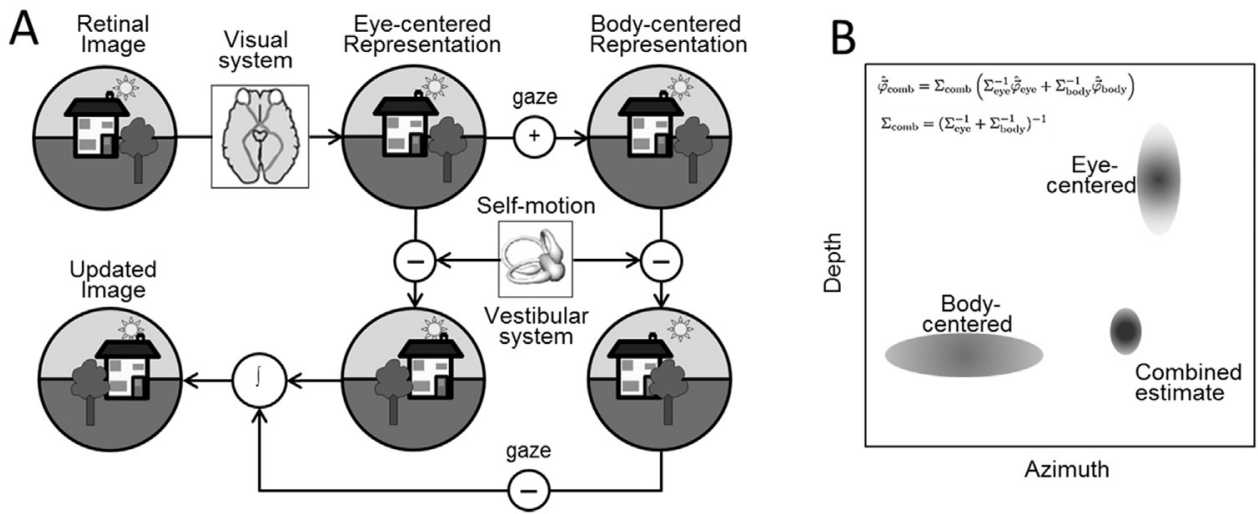

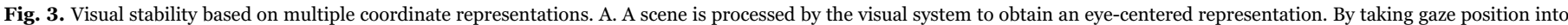

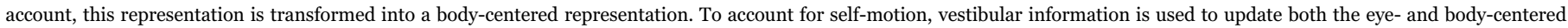

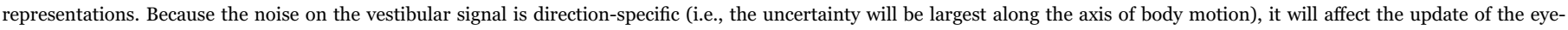

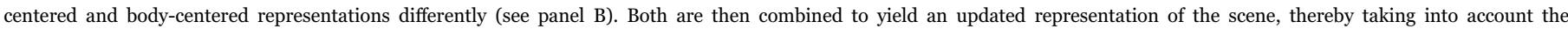

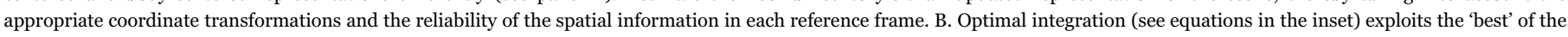
representations in the two reference frames.

(Avillac et al., 2005; Chang et al., 2009; Stricanne et al., 1996). It has been argued that this heterogeneity provides the parietal cortex with a mechanism to implicitly create multiple modes of representation at the population level, with each reference frame weighted and updated by sensorimotor input (Buchholz et al., 2013; Pouget et al., 2002). We think that this is what is probed in our behavioral experiment. In the following section we will ask the question whether we can probe these, or some of these, dynamic state estimates in the human brain.

\section{Cortical representations of self-motion remapping}

The combination of behavioral and computational work on eye movements provides evidence that the state estimator codes representations of the environment dynamically, in eye-centered coordinates. These eye-centered representations are supposed to be remapped, some even predictively, in connection with an intended or executed eye movement. Till now, various cortical and subcortical regions have been implicated in this eye-centered remapping, in both primates (Sommer and Wurtz, 2008) and humans (Medendorp, 2011). However, when the body is in motion, both the geometry of remapping changes, as well as the signals that are involved (see previous sections). This raises the question whether there is also neurophysiological evidence for remapping of targets while the body is in motion.

This question was recently addressed by Gutteling et al. (2015, 2016) using EEG. They reported neural evidence for remapping in the updating of state estimates during passively-induced body translations. In these experiments, subjects were asked to remember the location of a target, briefly presented in front or behind the fixation point before the start of a passively-induced whole body translation. During the translation, the fixation point remained either stationary to the world or stationary to the body. The subject was instructed to remember the location of the target relative to the world or to the body. After the motion, the memory update was assessed using a behavioral response. While the body motion, as detected by the vestibular system, should not affect the representation of body-fixed targets, it should interact with the representation of a world-centered target to update its location relative to the body. In the latter case, for veridical state estimation, an eye centered remapping mechanism must also take into account that objects behind and in front move in different directions relative to gaze; i.e. must take into account the geometry of motion parallax (Fig. 4A, Clemens et al., 2012; Medendorp et al., 2003; Tramper and Medendorp, 2015; Van Pelt and Medendorp, 2007).

Results show that the initial presentation of the visual target induced a reduction of alpha band power in contralateral parietooccipital areas. The subsequent motion of the body led to a reduction of alpha band power in central parietal areas extending to lateral parietotemporal areas, irrespective of whether the targets had to be memorized relative to world or body. This overall reduction in alpha band power reflects self-motion processing irrespective of the target updating. A similar suppression of alpha band activity in parietal areas has recently been reported for rotational self-motion (Gale et al., 2015).

When updating a world-fixed target, its internal representation shifts hemispheres (Fig. 4B), but only when subjects' behavioral responses suggested an update across the body midline (Gutteling and Medendorp, 2016). These shifts also follow the parallax geometry, i.e. the trans-hemispheric remapping depends on the remembered depth of the target relative to the fixation point (Gutteling et al., 2015). The strength of these remapping modulations correlated significantly with the quality of the state estimates (Fig. 4C), as represented by the behavioral updating errors of the individual subjects (Gutteling et al., 2015). These results can be interpreted as the involvement of parietal cortex in both self-motion estimation and the selective application of this motion information to maintaining states of target location as fixed in the world or fixed to the body.

It is important to point out that this evidence for remapping is based on the underlying hemispheric topography of alpha band activity. Because of the topographic organization, we could follow whether, at the neural populations level, activity changes hemispheres during the motion. If neurons are not topographically arranged along the dimensions of the reference frame they employ, we cannot distinguish this reference frame using this approach. This explains why we did not probe the body-centered representations in the alpha band activity.

\section{Decisions in motion}

Considering that target locations are represented as dynamic states, how does the brain select between these states in order to generate a motoric action? A large body of literature has shown that the decision making for action involves the coordinated integration of bottom-up sensory factors with top-down influences related to internal goals and rewards (Bisley and Goldberg, 2010; Schall et al., 2011; Sumner, 2011). For example, stimulus intensity and contrast influence saccadic reaction time (Marino et al., 2012) and anticipated biomechanical costs may affect the outcome of reach decisions (Cos et al., 2011).

Again, the vast majority of the extensive work on target selection has been done when the subject is stationary, neglecting the far more typical situation when the subject is in motion, like in driving or walking. New questions arise when research in decision making is broadened to conditions of self-motion, where vestibular signals play a 
A

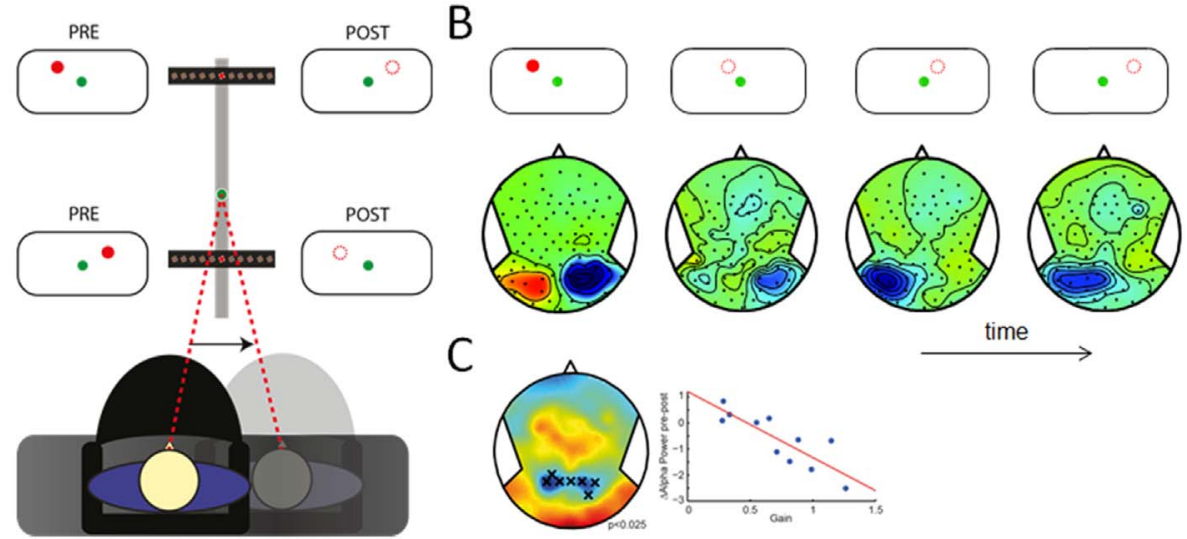

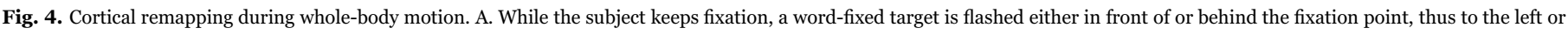

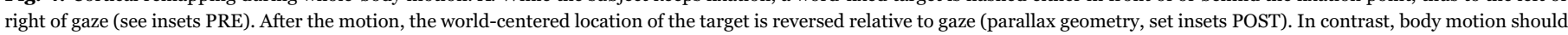

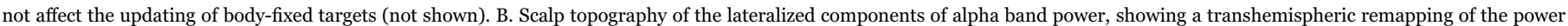

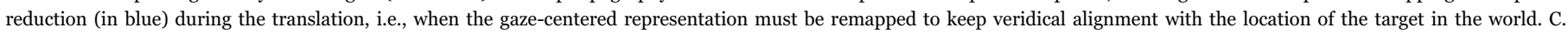
Alpha-band modulations correlate with updating performance across subjects.

key role.

The vestibular system has a profound influence on eye movements, as seen in the vestibular ocular reflex (Angelaki and Cullen, 2008; Paige et al., 1998). Brainstem circuits for saccades and vestibular quickphase responses converge in the burst generator (Scudder et al., 2002). Based on these findings, one could surmise that vestibular information could provide a highly potent bottom-up influence on decision-making. Alternatively, subjects may adopt a habitual top-down strategy to bias decisions based on predictions of upcoming motion. This requires the brain to build an internal model of the motion dynamics, which may involve vestibular signals (Prsa et al., 2015).

To dissociate between these hypotheses, we recently examined saccadic decision making while subjects were sinusoidally translated by a whole-body motion platform (Rincon-Gonzalez et al., 2016). During the motion, two visual targets were presented asynchronously but equidistantly on either side of the body stationary fixation point. Upon presentation, subjects had to look at one of these targets as quickly as possible (Fig. 5A). Using an adaptive psychometric procedure, we adjusted the time delay between the two target onsets until the subject selected both targets equally often as the goal of the saccade.

This balanced time delay - a measure of the choice bias - was determined for different phases of the motion in order to distinguish the effects of body acceleration and velocity on saccadic target selection. We reasoned that a low-level, bottom-up influence of vestibular information on decision-making affects saccade choice at peak acceleration (when velocity is zero), but not at peak velocity (when acceleration is zero). In contrast, if the brain uses top-down information, saccadic choice may be more related to the direction of motion, i.e. to velocity-based signals.

Results show that acceleration, but not velocity, affects target selection for saccades (Fig. 5B). Subjects preferred to look at targets in the direction of the acceleration - the leftward target was preferred when the sled accelerated to the left, and vice versa. Saccadic reaction times were also reliably shorter to targets in the acceleration direction, which is a further reflection of the selection bias (Fig. 5C).

These results identify bottom-up acceleration, derived directly from the otolith system, as the main dynamic factor influencing saccadic decisions. As a neurophysiological explanation, it is plausible that vestibular information from the otolith organs modulates superior colliculus (SC) activity during our saccadic decision paradigm (Hepp et al., 1993), favoring development of a saccade-related burst in one SC versus the other. An alternative possibility is that the vestibular signals modulate activity in cortical areas that have been implicated in saccade planning, such as the lateral intraparietal area (Shinder and Taube, 2010) or the frontal eye fields (Fukushima, 1997). As mentioned above,
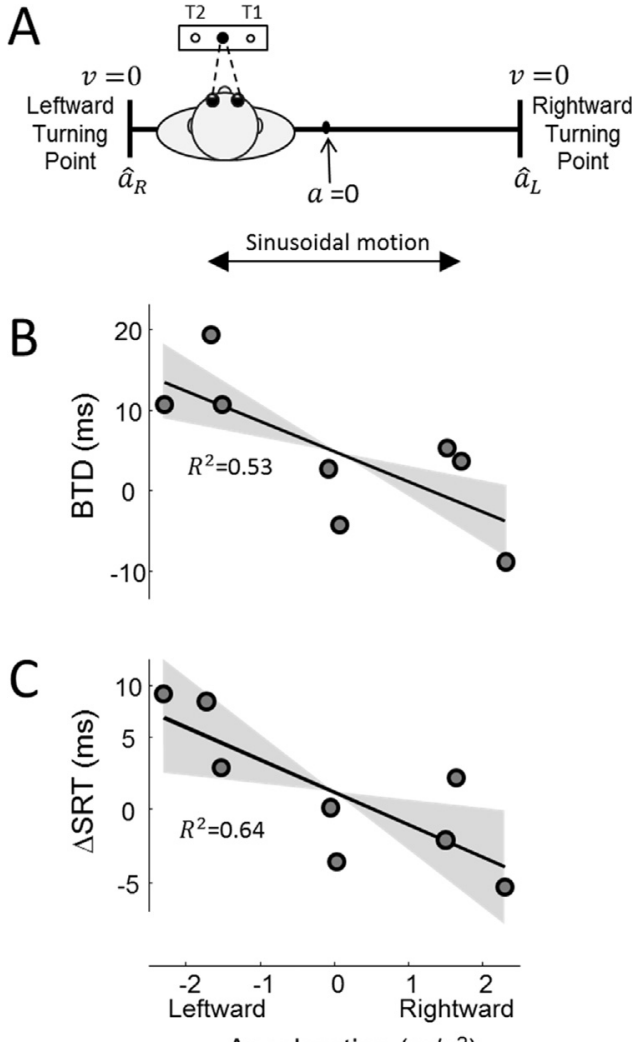

Acceleration $\left(\mathrm{m} / \mathrm{s}^{2}\right)$

Fig. 5. Decisions in motion. A. Subjects were translated sinusoidally while fixating a central, body-fixed light. At different phases of the motion, two peripheral lights were presented with a small stimulus onset asynchrony; subjects had to look as fast as possible to one of these lights. Acceleration peaks at the turning points, while velocity is zero, and acceleration is zero midway, while velocity peaks. B. Balance time delay as a function of acceleration. C. Reaction time difference between rightward and leftward saccades as a function of acceleration.

there are pathways that transmit vestibular signals to parietal cortex and frontal cortex, which could potentially bias processing in the SC, but this has not been tested.

While the results above concern the vestibular contribution to the decision on where to look, i.e. whether to look leftward or rightward, decisions in motion may also have a when component, i.e. the initiation of the act (Haggard, 2008). We addressed this question recently by examining the phase relationships that characterize the coordination of 

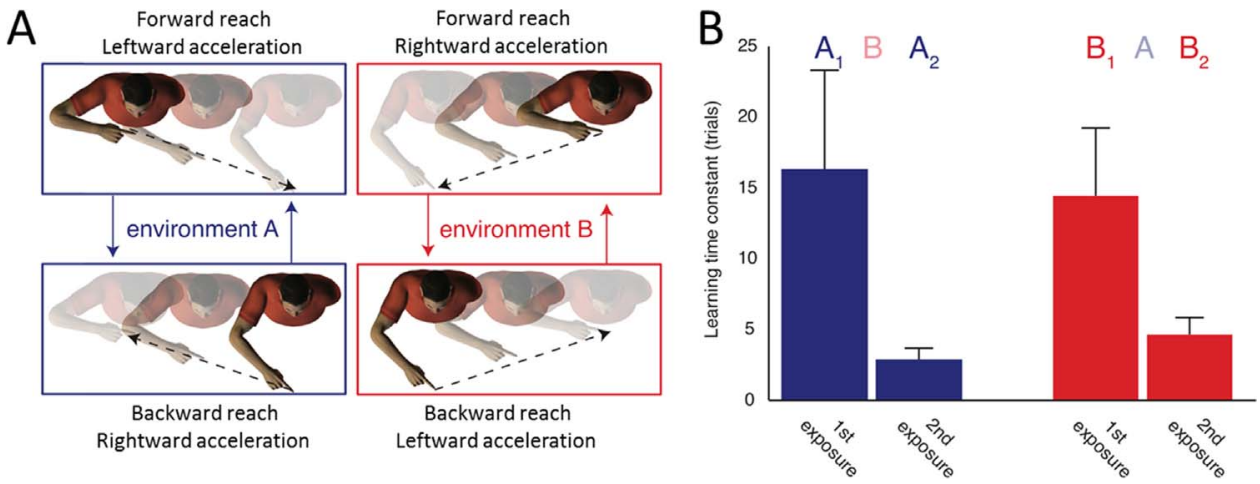

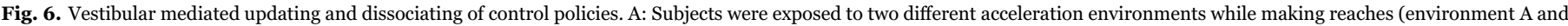

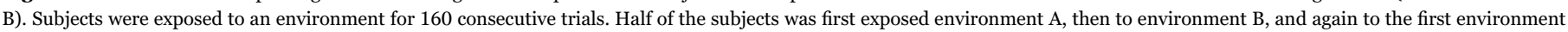

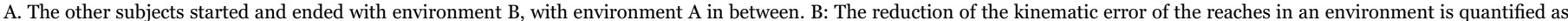

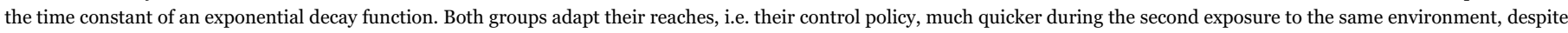
the opposite environment being in between.

arm-reaching movements with passively-induced whole-body motion (Bakker et al., 2015). Experimentally, we observed a stable in-phase relationship when reaches are made between targets that are aligned parallel to the body motion. In fact, when we entrained subjects first with an anti-phase relationship (i.e. they moved to the left target while the whole body motion was to the right), they quickly fell back toward the in-phase relationship after the entrainment. These results cannot be explained by optimality criteria such as minimizing energy cost, which predict a stable anti-phase relationship. It is possible that neural constraints and predictive mechanisms drive this coordination, perhaps based on gaze and vestibular signals, but it would require further studies to find out.

These new results emphasize the impact of vestibular information on motor decision making, extending the importance of this sensory modality beyond its known role in reflexive responses and spatial orientation. It would be interesting, in future work, to address whether and how bottom-up and/or top-down influences of acceleration influence saccadic decisions when the head is free to move, or bias target selection of reaching movements, or affect the choice of which hand to use.

\section{Learning and recall}

It has been argued that the processes of action selection and specification are performed in parallel. The brain specifies multiple response programs in parallel up to the level of motor preparation (Cisek and Kalaska, 2010), while a variety of biasing influences cause one specific action to prevail, including vestibular influences. Can vestibular signals also be used as feedback signals to correct an ongoing action, or to select and update control policies?

Compared to the eyes, which have negligible mass, the arm has a substantial mass and inertia, which need to be incorporated into the control policy or internal model of a reaching movement executed under whole body motion. The feedback control policy must take into account the inertial forces induced by the body motion, which can be derived from the vestibular signals. Indeed, it has been shown that subjects can process vestibular signals quite accurately in the online control of reaching movements during slow passive body rotations (see Blouin et al., 2015, for review), suggesting that the control policy does take vestibular feedback into account. Other studies have shown that the brain accounts for Coriolis torques on the limb generated during active torso rotation (Pigeon et al., 2013), indicating that the control policy incorporates a model of expected torques related to the body rotation. Furthermore, the brain adapts its control policy to the Coriolis torques felt during reaches made in rotating environments (see Lackner and Dizio, 2005, for review). Similarly, we showed that human subjects adapt their control policy for reaching under passive lateral translation on a vestibular platform, inducing inertial forces on the arm (Sarwary et al., 2013).

While it is clear that our brain is able to form new internal models and update existing ones, there is still debate on how the correct internal model can be accessed and recalled, without interfering with other stored models (Howard et al., 2013). We seem capable of remembering many different types of actions, without having the one action confused with, or affected by the other. But what allows this flexibility is much less clear. Laboratory experiments have shown that the control policy of one task is lost or cannot be recalled after learning an opposing task, pointing to both anterograde and retrograde interference effects (Caithness et al., 2004). However, if context can be used to distinguish tasks, it appears that such interference is reduced, permitting simultaneous learning and recall of multiple internal models (Howard et al., 2013). It is important to note though that the context should be of special nature; e.g. simple declarative cues like color or haptic cues do not work (Cothros et al., 2008; Gandolfo et al., 1996). It appears that contextual cues should be part of the sensorimotor loop in order to be effective in learning and later recall.

If the notion that contextual cues need to be part of the sensorimotor loop is accepted, vestibular information could be an effective cue to keep multiple internal models separated. During self-motion, vestibular information is needed for online reach corrections based on the current control policy, but may also serve as a contextual cue to update that same control policy.

We tested this recently, asking subjects to reach while their body was passively translated laterally (Sarwary et al., 2013). We switched the coupling between the direction of a reaching movement (forwardbackward) and the direction of whole-body motion (leftward-rightward) every 160 trials (see Fig. 6A). When exposed for the second time to the same coupling between lateral motion and reach direction, with the opposite coupling in between, subjects were able to readily recall the earlier learned dynamics, and act as if the coupling was still known (Fig. 6B).

This suggests that the vestibular system plays an important role in coding and decoding multiple control policies. The vestibular system may not only assist in the estimation of the imposed forces, but also provide a contextual signal reducing interference between different control policies. These results indicate the importance of investigating motor learning in rich sensory contexts, including vestibular signals, that more closely match the natural environment in which we move than the classic reach adaptation paradigm with the upper body restrained.

\section{Conclusion}

This review emphasizes the impact of vestibular information on 
cognition, extending the importance of this sensory modality beyond its known role in reflexive responses and spatial orientation. We have shown how the vestibular system plays a key role in estimating the state of the world and body, in tasks ranging from updating spatial targets, to decision making, to reaching in accelerating environments. All these results indicate the importance of investigating the sensorimotor system in rich sensory environments in order to appreciate the sophistication of the estimation and control systems implemented in the brain. The neuro-computational mechanisms that characterize these systems can be formally described in terms of inference, coordinate representations and learning from prediction errors. We believe that these concepts are not unique to understanding the sensorimotor system, but rather point to general principles that are widely involved in the classically-defined cognitive faculties, including language, memory, and consciousness. Understanding how sensorimotor acts come about during body motion is also particularly relevant for real-world behavior, such as in sports, car-driving and all kinds of human-human interactions. Despite all these new insights, we have also listed various questions that require further study. It would be particularly interesting to set up neurophysiological investigations into precisely how the results we described are mediated.

\section{Acknowledgments}

We thank dr. Leonie Oostwoud-Wijdenes for her valuable comments on this manuscript. This work is supported the European Research Council (EU-ERC-283567), EU-FP7-FET grant (SpaceCog 600785), and the Netherlands Organization for Scientific Research (NWO-VICI: 453-11-001).

\section{References}

Angelaki, D.E., Cullen, K.E., 2008. Vestibular system: the many facets of a multimodal sense. Annu. Rev. Neurosci. 31, 125-150. http://dx.doi.org/10.1146/ annurev.neuro.31.060407.125555.

Angelaki, D.E., McHenry, M.Q., Dickman, J.D., Newlands, S.D., Hess, B.J., 1999. Computation of inertial motion: neural strategies to resolve ambiguous otolith information. J. Neurosci. 19, 316-327.

Angelaki, D.E., Shaikh, A.G., Green, A.M., Dickman, J.D., 2004. Neurons compute internal models of the physical laws of motion. Nature 430, 560-564. http:// dx.doi.org/10.1038/nature02754.

Atsma, J., Maij, F., Koppen, M., Irwin, D.E., Medendorp, W.P., 2016. Causal inference for spatial constancy across saccades. PLoS Comput. Biol. 12, e1004766. http:// dx.doi.org/10.1371/journal.pcbi.1004766.

Avillac, M., Denève, S., Olivier, E., Pouget, A., Duhamel, J.-R., 2005. Reference frames for representing visual and tactile locations in parietal cortex. Nat. Neurosci. 8, 941-949. http://dx.doi.org/10.1038/nn1480.

Bakker, R.S., Selen, L.P.J., Medendorp, W.P., 2015. Stability of phase relationships while coordinating arm reaches with whole body motion. PLoS One 10, e0146231. http:// dx.doi.org/10.1371/journal.pone.0146231.

Berniker, M., Kording, K., 2008. Estimating the sources of motor errors for adaptation and generalization. Nat. Neurosci. 11, 1454-1461. http://dx.doi.org/10.1038/ nn.2229.

Bisley, J.W., Goldberg, M.E., 2010. Attention, intention, and priority in the parietal lobe. Annu. Rev. Neurosci. 33, 1-21. http://dx.doi.org/10.1146/annurev-neuro-060909152823.

Blouin, J., Bresciani, J.-P., Guillaud, E., Simoneau, M., 2015. Prediction in the vestibular control of arm movements. Multisens. Res. 28, 487-505.

Buchholz, V.N., Jensen, O., Medendorp, W.P., 2013. Parietal oscillations code nonvisual reach targets relative to gaze and body. J. Neurosci. 33, 3492-3499. http:// dx.doi.org/10.1523/JNEUROSCI.3208-12.2013.

Caithness, G., Osu, R., Bays, P., Chase, H., Klassen, J., Kawato, M., Wolpert, D.M., Flanagan, J.R., 2004. Failure to consolidate the consolidation theory of learning for sensorimotor adaptation tasks. J. Neurosci. 24, 8662-8671. http://dx.doi.org/ 10.1523/JNEUROSCI.2214-04.2004.

Chang, S.W.C., Papadimitriou, C., Snyder, L.H., 2009. Using a compound gain field to compute a reach plan. Neuron 64, 744-755. http://dx.doi.org/10.1016/ j.neuron.2009.11.005.

Chemero, A., 2009. Radical Embodied Cognitive Science. MIT Press.

Cisek, P., Kalaska, J.F., 2010. Neural mechanisms for interacting with a world full of action choices. Annu. Rev. Neurosci. 33, 269-298. http://dx.doi.org/10.1146/ annurev.neuro.051508.135409.

Clark, A., 2010. Coupling, constitution and the cognitive kind: a reply to Adam and Aizawa. In: Menary, R. (Ed.), The Extended Mind. MIT Press, Cambridge, 81-99.

Clemens, I.A.H., De Vrijer, M., Selen, L.P.J., Van Gisbergen, J.A.M., Medendorp, W.P., 2011. Multisensory processing in spatial orientation: an inverse probabilistic approach. J. Neurosci. 31, 5365-5377. http://dx.doi.org/10.1523/ JNEUROSCI.6472-10.2011.

Clemens, I.A.H., Selen, L.P.J., Koppen, M., Medendorp, W.P., 2012. Visual stability across combined eye and body motionJ. Vis., 12. http://dx.doi.org/10.1167/12.12.8.

Clemens, I.A.H., Selen, L.P.J., Pomante, A., MacNeilage, P.R., Medendorp, W.P., 2017. Eye-movements in darkness modulate self-motion perception. eNeuro. http:// dx.doi.org/10.1523/ENEURO.0211-16.2016.

Cos, I., Bélanger, N., Cisek, P., 2011. The influence of predicted arm biomechanics on decision making. J. Neurophysiol. 105, 3022-3033. http://dx.doi.org/10.1152/ jn.00975.2010.

Cothros, N., Wong, J., Gribble, P.L., 2008. Distinct haptic cues do not reduce interference when learning to reach in multiple force fields. PLoS One 3, e1990. http:// dx.doi.org/10.1371/journal.pone.0001990.

Descartes, R., 1644. Principia philosophiae.

Diedrichsen, J., Shadmehr, R., Ivry, R.B., 2010. The coordination of movement: optimal feedback control and beyond. Trends Cogn. Sci. 14, 31-39. http://dx.doi.org/ 10.1016/j.tics.2009.11.004

Drugowitsch, J., DeAngelis, G.C., Klier, E.M., Angelaki, D.E., Pouget, A., 2014. Optimal multisensory decision-making in a reaction-time task. Elife, 3. http://dx.doi.org/ 10.7554/eLife.03005.

Ernst, M.O., Bülthoff, H.H., 2004. Merging the senses into a robust percept. Trends Cogn. Sci. 8, 162-169. http://dx.doi.org/10.1016/j.tics.2004.02.002.

Faisal, A., Selen, L., Wolpert, D., 2008. Noise in the nervous system. Nat. Rev. Neurosci..

Fetsch, C.R., DeAngelis, G.C., Angelaki, D.E., 2013. Bridging the gap between theories of sensory cue integration and the physiology of multisensory neurons. Nat. Rev. Neurosci. 14, 429-442. http://dx.doi.org/10.1038/nrn3503.

Frenz, H., Lappe, M., 2005. Absolute travel distance from optic flow. Vis. Res. 45, 1679-1692. http://dx.doi.org/10.1016/j.visres.2004.12.019.

Fukushima, K., 1997. Corticovestibular interactions: anatomy, electrophysiology, and functional considerations. Exp. Brain Res. 117, 1-16.

Gale, S., Prsa, M., Schurger, A., Gay, A., Paillard, A., Herbelin, B., Guyot, J.-P., Lopez, C., Blanke, O., 2015. Oscillatory neural responses evoked by natural vestibular stimuli in humans. J. Neurophysiol. 00153, 2015. http://dx.doi.org/10.1152/jn.00153.2015, jn.00153.2015.

Gandolfo, F., Mussa-Ivaldi, F.A., Bizzi, E., 1996. Motor learning by field approximation. Proc. Natl. Acad. Sci. USA 93, 3843-3846.

Gibson, J., 1966. The Senses Considered as Perceptual Systems. Hougton Mifflin, Boston, MA.

Gu, Y., Angelaki, D.E., Deangelis, G.C., 2008. Neural correlates of multisensory cue integration in macaque MSTd. Nat. Neurosci. 11, 1201-1210. http://dx.doi.org/ 10.1038/nn.2191.

Gutteling, T.P., Medendorp, W.P., 2016. Role of Alpha-band oscillations in spatial updating across whole body motion. Front. Psychol., 7. http://dx.doi.org/10.3389/ fpsyg.2016.00671.

Gutteling, T.P., Selen, L.P.J., Medendorp, W.P., 2015. Parallax-sensitive remapping of visual space in occipito-parietal alpha-band activity during whole-body motion. J. Neurophysiol. 113, 1574-1584. http://dx.doi.org/10.1152/jn.00477.2014.

Haggard, P., 2008. Human volition: towards a neuroscience of will. Nat. Rev. Neurosci. 9 , 934-946. http://dx.doi.org/10.1038/nrn2497+.

Harris, C.M., Wolpert, D.M., 1998. Signal-dependent noise determines motor planning. Nature 394 (6695), 780-784.

Hepp, K., Van Opstal, A.J., Straumann, D., Hess, B.J., Henn, V., 1993. Monkey superior colliculus represents rapid eye movements in a two-dimensional motor map. J. Neurophysiol. 69, 965-979.

Holst, N., Mittelstaedt, D.H., 1950. The principle of Reafference: interactions between the central nervous System and the peripheral organs. Naturwissenschaften, 37.

Howard, I.S., Wolpert, D.M., Franklin, D.W., 2013. The effect of contextual cues on the encoding of motor memories. J. Neurophysiol. 109, 2632-2644. http://dx.doi.org/ 10.1152/jn.00773.2012.

Jones, K.E., Hamilton, A.F., Wolpert, D.M., 2002. Sources of signal-dependent noise during isometric force production. J. Neurophysiol. 88, 1533-1544.

Kawato, M., 1999. Internal models for motor control and trajectory planning. Curr. Opin. Neurobiol. 9, 718-727.

Körding, K.P., Beierholm, U., Ma, W.J., Quartz, S., Tenenbaum, J.B., Shams, L., 2007. Causal inference in multisensory perception. PLoS One 2, e943. http://dx.doi.org/ 10.1371/journal.pone.0000943.

Körding, K.P., Wolpert, D.M., 2004. Bayesian integration in sensorimotor learning. Nature 427, 244-247. http://dx.doi.org/10.1038/nature02169.

Lackner, J.R., Dizio, P., 2005. Motor control and learning in altered dynamic environments. Curr. Opin. Neurobiol. 15, 653-659. http://dx.doi.org/10.1016/ j.conb.2005.10.012.

Laurens, J., Droulez, J., 2007. Bayesian processing of vestibular information. Biol. Cybern. 96, 389-404. http://dx.doi.org/10.1007/s00422-006-0133-1.

Laurens, J., Meng, H., Angelaki, D.E., 2013. Computation of linear acceleration through an internal model in the macaque cerebellum. Nat. Neurosci. 16, 1701-1708. http:// dx.doi.org/10.1038/nn.3530.

Marino, R.A., Levy, R., Boehnke, S., White, B.J., Itti, L., Munoz, D.P., 2012. Linking visual response properties in the superior colliculus to saccade behavior. Eur. J. Neurosci. 35, 1738-1752. http://dx.doi.org/10.1111/j.1460-9568.2012.08079.x.

McGuire, L.M.M., Sabes, P.N., 2009. Sensory transformations and the use of multiple reference frames for reach planning. Nat. Neurosci. 12, 1056-1061. http:// dx.doi.org/10.1038/nn.2357.

Medendorp, W.P., 2011. Spatial constancy mechanisms in motor control. Philos. Trans. R. Soc. Lond. B. Biol. Sci. 366, 476-491. http://dx.doi.org/10.1098/rstb.2010.0089.

Medendorp, W.P., Tweed, D.B., Crawford, J.D., 2003. Motion parallax is computed in the updating of human spatial memory. J. Neurosci. 23, 8135-8142. 
Merfeld, D.M., Zupan, L., Peterka, R.J., 1999. Humans use internal models to estimate gravity and linear acceleration. Nature 398, 615-618. http://dx.doi.org/10.1038/ 19303.

Mittelstaedt, H., 1992. Somatic versus vestibular gravity reception in Man. Ann. N. Y. Acad. Sci. 656, 124-139. http://dx.doi.org/10.1111/j.1749-6632.1992.tb25204.x.

Munuera, J., Morel, P., Duhamel, J.-R., Deneve, S., 2009. Optimal sensorimotor control in eye movement sequences. J. Neurosci. 29, 3026-3035. http://dx.doi.org/ 10.1523/JNEUROSCI.1169-08.2009.

Niemeier, M., Crawford, J.D., Tweed, D.B., 2003. Optimal transsaccadic integration explains distorted spatial perception. Nature 422 (6927), 76-80.

Niemeier, M., Crawford, J.D., Tweed, D.B., 2007. Optimal inference explains dimensionspecific contractions of spatial perception. Exp. Brain Res. 179, 313-323. http:// dx.doi.org/10.1007/s00221-006-0788-9.

Oostwoud Wijdenes, L., Marshall, L., Bays, P.M., 2015. Evidence for optimal integration of visual feature representations across saccades. J. Neurosci. 35, 10146-10153. http://dx.doi.org/10.1523/JNEUROSCI.1040-15.2015.

Paige, G.D., Telford, L., Seidman, S.H., Barnes, G.R., 1998. Human vestibuloocular reflex and its interactions with vision and fixation distance during linear and angular head movement. J. Neurophysiol. 80, 2391-2404.

Pigeon, P., Dizio, P., Lackner, J.R., 2013. Immediate compensation for variations in selfgenerated Coriolis torques related to body dynamics and carried objects. $\mathrm{J}$. Neurophysiol. 110, 1370-1384. http://dx.doi.org/10.1152/jn.00104.2012.

Pouget, A., Deneve, S., Duhamel, J.-R., 2002. A computational perspective on the neural basis of multisensory spatial representations. Nat. Rev. Neurosci. 3, 741-747. http://dx.doi.org/10.1038/nrn914.

Prevosto, V., Graf, W., Ugolini, G., 2009. Posterior parietal cortex areas MIP and LIPv receive eye position and velocity inputs via ascending preposito-thalamo-cortical pathways. Eur. J. Neurosci. 30, 1151-1161. http://dx.doi.org/10.1111/j.14609568.2009.06885.x.

Prsa, M., Jimenez-Rezende, D., Blanke, O., 2015. Inference of perceptual priors from path dynamics of passive self-motion. J. Neurophysiol. 113, 1400-1413. http:// dx.doi.org/10.1152/jn.00755.2014.

Rincon-Gonzalez, L., Selen, L.P.J., Halfwerk, K., Koppen, M., Corneil, B.D., Medendorp, W.P., 2016. Decisions in motion: vestibular contributions to saccadic target selection. J. Neurophysiol. 116, 977-985. http://dx.doi.org/10.1152/ jn.01071.2015.

Sarwary, A.M.E., Selen, L.P.J., Medendorp, W.P., 2013. Vestibular benefits to task savings in motor adaptation. J. Neurophysiol. 110, 1269-1277. http://dx.doi.org/ 10.1152/jn.00914.2012.

Schall, J.D., Purcell, B.A., Heitz, R.P., Logan, G.D., Palmeri, T.J., 2011. Neural mechanisms of saccade target selection: gated accumulator model of the visualmotor cascade. Eur. J. Neurosci. 33, 1991-2002. http://dx.doi.org/10.1111/j.14609568.2011.07715.x.

Shadmehr, R., Krakauer, J.W., 2008. A computational neuroanatomy for motor control. Exp. Brain Res. 185, 359-381. http://dx.doi.org/10.1007/s00221-008-1280-5.

Shadmehr, R., Smith, M. a., Krakauer, J.W., 2010. Error correction, sensory prediction, and adaptation in motor control. Annu. Rev. Neurosci. 33, 89-108. http:// dx.doi.org/10.1146/annurev-neuro-060909-153135.

Shinder, M.E., Taube, J.S., 2010. Differentiating ascending vestibular pathways to the cortex involved in spatial cognition. J. Vestib. Res. 20, 3-23. http://dx.doi.org/ 10.3233/VES-2010-0344.

Sommer, M.A., Wurtz, R.H., 2008. Brain circuits for the internal monitoring of movements. Annu. Rev. Neurosci. 31, 317-338. http://dx.doi.org/10.1146/ annurev.neuro.31.060407.125627.

Stricanne, B., Andersen, R. a., Mazzoni, P., 1996. Eye-centered, head-centered, and intermediate coding of remembered sound locations in area LIP. J. Neurophysiol. 76, 2071-2076. http://dx.doi.org/10.1021/je9001366.

Sumner, P., 2011. Determinants of saccadic latency. In: Liversedge, S., Gilchrist, I., Everling, S. (Eds.), The Oxford Handbook of Eye Movements. Oxford University Press, Oxford.

ter Horst, A.C., Koppen, M., Selen, L.P.J., Medendorp, W.P., 2015. Reliability-based weighting of visual and vestibular cues in displacement estimation. PLoS One 10, e0145015. http://dx.doi.org/10.1371/journal.pone.0145015.

Todorov, E., 2010. Parallels between sensory and motor information processing. In: Gazzaniga (Ed.), The Cognitive Neurosciences.

Todorov, E., Jordan, M.I., 2002. Optimal feedback control as a theory of motor coordination. Nat. Neurosci. 5, 1226-1235. http://dx.doi.org/10.1038/nn963.

Tramper, J.J., Medendorp, W.P., 2015. Parallel updating and weighting of multiple spatial maps for visual stability during whole-body motion. J. Neurophysiol. 114 http://dx.doi.org/10.1152/jn.00576.2015.

Trousselard, M., Barraud, P., Nougier, V., Raphel, C., Cian, C., 2004. Contribution of tactile and interoceptive cues to the perception of the direction of gravity. Brain Res. Cogn. Brain Res. 20, 355-362. http://dx.doi.org/10.1016/ j.cogbrainres.2004.03.008.

Tweed, D., 2003. Microcosms of the Brain: What Sensorimotor Systems Reveal about the Mind. Oxford University Press.

Van Pelt, S., Medendorp, W.P., 2007. Gaze-centered updating of remembered visual space during active whole-body translations. J. Neurophysiol. 97, 1209-1220. http://dx.doi.org/10.1152/jn.00882.2006.

Vaziri, S., Shadmehr, R., 2006. Why does the brain predict sensory consequences of oculomotor commands? Optim. Integr. Predict. Actual Sens. Feedback 26, 4188-4197. http://dx.doi.org/10.1523/JNEUROSCI.4747-05.2006.

Von Helmholtz, H., 1867. Handbuch der physiologischen Optik. Voss, Leipzig.

Warren, W.H., Hannon, D.J., 1990. Eye movements and optical flow. J. Opt. Soc. Am. A 7, 160-169.

Wertheim, A.H., Mesland, B.S., Bles, W., 2001. Cognitive suppression of tilt sensations during linear horizontal self-motion in the dark. Perception 30, 733-741.

Wolpert, D.M., Ghahramani, Z., Flanagan, J.R., 2001. Perspectives and problems in motor learning. TRENDS Cogn. Sci., 5.

Yuste, R., 2015. From the neuron doctrine to neural networks. Nat. Rev. Neurosci. 16, 487-497. http://dx.doi.org/10.1038/nrn3962. 